\section{SCOPE AND LIMITATIONS OF INFRA-RED MEASUREMENTS IN CHEMISTRY*}

\author{
By DR. H. W. THOMPSON \\ University of Oxford
}

$\mathrm{F}$ OR some years infra-red spectroscopy has been a valuable research tool in pure chemistry, particularly in problems of molecular structure and the deduction of molecular data. Its value in industrial work, both for routine and research measurements, is now becoming fully appreciated, and its usefulness in general organic chemistry may soon at least equal that of ultra-violet spectroscopy. It is therefore opportune to consider its uses and limitations.

The infra-red may be said to extend between wavelengths of about $1 \mu$ and $10^{4} \mu$. This corresponds to a range of frequencies $3 \times 10^{14}$ to $3 \times 10^{10}$ per second, or $10^{4}$ to 1 wave numbers. A small region from the visible up to $1 \cdot 2 \mu$ can be studied photographically, using conventional spectrographs with glass prisms or finely ruled diffraction gratings. Between $1 \mu$ and $25 \mu$, prism spectrometers are most often used, in which the prism is made of quartz, fluorite, rock salt or potassium halides. Since large natural crystals of these materials are growing scarce, their production recently in large blocks by cooling the molten salts is important. Diffraction gratings have to be used at wave-lengths beyond $25 \mu$, although they are also used at shorter wave-lengths if higher resolving power is required. In order to obtain a greater concentration of energy and avoid its dissipation among many spectral orders, these 'echelette' gratings are ruled with specially shaped grooves so that reflexion occurs at favoured angles. The infra-red radiation is usually detected by means of thermo-electric devices. Striking advances have recently been made by the development of very sensitive vacuum thermocouples and voltage amplifiers, thus leading to an increase in both sensitivity and stability of the galvanometric systems, and to the construction of automatically recording spectrometers. In this way, infra-red technique has ceased to be a delicate research method and has become more easily applicable by non-specialists.

Infra-red emission spectra are rarely intense enough for measurement, and we are nearly always concerned with the absorption spectra. In this spectral range, the quanta of energy absorbed cause the molecules to rotate and vibrate. In order to absorb rotational quanta, a molecule must have a permanent dipole moment. Vibrational quanta are absorbed if the particular vibration involves a changing molecular dipole moment. Thus, although homonuclear molecules like hydrogen or nitrogen do not absorb vibrational quanta, methane can do so, since it can perform an unsymmetrical nuclear vibration which produces an electric moment.

Rotational quanta are nearly always small, and the pure rotational spectra therefore lie at very long wave-lengths, between about $10^{2} \mu$ and $10^{4} \mu$, or 1-100 wave numbers. Little is so far known about them, mainly owing to technical difficulties in studying this region, but also because of their complexity with all except diatomic molecules. In the latter case, the spacing between rotational lines leads

* Substance of the Tilden Lecture, delivered before the Chemical Society on January 20. to the molecular moment of inertia and hence to the bond-length.

Vibrational quanta are larger, the fundamentals varying from about $100 \mathrm{~cm} .^{-1}$ to $4,000 \mathrm{~cm}$. ${ }^{1}$. The fundamentals, lower harmonics, and combinations are therefore absorbed between about $100 \mu$ and $1 \mu$. Changes in rotational energy usually accompany the absorption of vibrational quanta, and with gases or vapours this gives rise to a fine structure of the vibration bands, the precise details of which are of great significance. With liquids and solutions, the intermolecular influences lead to the disappearance of this rotational structure.

A rigid non-linear molecule has $(3 n-6)$ normal vibrations, and a linear molecule $(3 n-5)$. With simple symmetrical molecules like carbon dioxide, water or ethylene, it is possible to specify in a general way the geometrical form of these vibrations. They ean sometimes be described as 'breathing', 'rocking', 'bending' or 'twisting' motions, and it is also sometimes convenient to differentiate between valency vibrations and deformational modes according as the motions of nuclei are along or perpendicular to the valency bonds. If a molecule has some degree of symmetry, its vibrations can also be characterized with reference to each element of symmetry, and by the way in which the electric moment changes with reference to these symmetry axes. Thus, the 'breathing' vibration of carbon dioxide is symmetrical with respect to the centre of symmetry - the carbon atom; and the other stretching vibration is antisymmetrical. In the latter case, the change of electric moment occurs in a direction parallel to the molecular axis, which is an axis of symmetry; in the deformational mode of carbon dioxide, however, the change in moment is perpendicular to the molecular axis. When the molecule has several planes, or other elements of symmetry, a given vibration may be symmetrical or antisymmetrical with respect to each. In the same way the total change in electric moment in different vibrations will vary in magnitude and in direction with respect to the symmetry elements. Some vibrations, for example, the totally symmetrical modes of ethylene, will involve no change in electric moment, and will not be absorbed as fundamentals in the infra-red. In other cases, the intensities of absorption will vary as the position-rate of change of electric moment during the vibration. For these reasons, intensities, taken alone, are no rigid guide in the allocation of observed frequencies to the fundamental modes. Many semi-empirical rules have to be applied, such as the persistence of some magnitudes through a related series of compounds, the fact that motions of lighter nuclei will usually have higher frequencies than those of heavier ones, and the com. parison of spectra of isotopic molecules, particularly those containing hydrogen and deuterium.

For certain purposes, a correct assignment of the fundamental frequencies is essential. The Rajnan spectrum provides valuable additional data. Here, vibrations are active if they involve a changing molecular polarizability. It therefore happens that frequencies which are not found in the infra-red spectrum may appear in the Raman spectrum, and vice versa. Raman frequencies also differ in their degree of polarization, which may enable us to allocate them more precisely to different types of vibration. Some oscillations, such as the twisting mode of ethylene, are inactive in both Raman and infra-red spectrum. These can sometimes be determined from fluorescence data, or from a comparison 
of the measured specific heat with that calculated statistically using all the known frequencies.

The other important guide in assigning the vibration frequencies is the analysis of the rotational structure of the infra-red absorption bands. If the molecule has some symmetry, this rotational structure differs according to the direction in which the electric moment changes with reference to the axes of symmetry. The. differences are often so characteristic that inspection may serve to allocate the vibration to a particular symmetry class. Detailed analysis of the spacings between the rotational lines may simultaneously lead to the moments of inertia and hence to knowledge about the molecular structure, and much information about simple molecules obtained in this way is more accurate than by any other method.

Such rotational analyses are limited, however, to molecules which are $(a)$ volatile enough to be studied as vapours, $(b)$ small enough to have low moments of inertia and resolvable structure, and (c) have some symmetry. These restrictions are severe, and most molecules with which the chemist has to deal are excluded. Even if $(b)$ and $(c)$ do not apply, however, useful information can often be obtained. Thus, if there is some symmetry but the moments of inertia are large, the envelope of the rotational structure, or band contour, can be measured. This contour, like the rotational structure itself, varies characteristically with the direction in which the electric moment changes during the vibration with reference to the axis of symmetry. By measurement of the spacing between sub-maxima in the band contour, moments of inertia can be roughly determined or an assumed molecular structure confirmed. If the molecule is asymmetrical, the rotational fine structure of bands is very complex, and although this class of molecules includes such simple structures as water, hydrogen sulphide, ethylene, and formaldehyde, few satisfactory analyses have so far been achieved. Here again, some progress can be made by measuring the band contours. These differ according as the direction of change of electric moment is parallel to the least, middle or greatest axis of inertia. In practice, hybrid contours are to be expected, since the change of electric moment will have components along more than one of these axes; but in many cases the contours are surprisingly simple, and not only help in assigning the frequencies but also serve to confirm assumed molecular structures.

It must be emphasized that even when all three principal moments of inertia of a molecule are known, the whole structure often cannot be deduced, since there are more than three bond-lengths and angles. One or more of the latter must then be assumed in order to determine the others. When data are available for a series of related molecules such as the methyl halides, self-consistency in the whole group may be a useful guide. In other cases, comparison of isotopic' molecules is valuable, particularly those containing hydrogen and deuterium.

Another serious difficulty in analysing rotational structures and contours results from perturbation of energy-levels due to interaction of vibration with rotation, which tends to be specific. On the other hand, incompletely resolved or partially unexplained rotational structures often serve to decide between alternative molecular configurations. In this way hydrogen peroxide has been shown to be non-planar, and formic acid to have a cis rather than trans structure.
When molecules are involatile, or large, only the vibration frequencies may be determinable. If all the frequencies are known, the specific heats can be calculated, using the results of statistical mechanics which relate them to the partition function. Discrepancies in some cases between the calculated and measured values have been correlated with the phenomenon of restricted rotation about bonds in organic molecules. Estimation of the potential onergy barriers restricting such internal rotation in series of molecules have now been made and throw some light on the nature of the forces between nonbonded atoms in molecules. If in addition to the vibration frequencies, the moments of inertia are also known, the free energy and entropy can be calculated; and provided other thermochemical data are avail. able, so that changes in zero-point energy can be calculated, equilibrium constants can be calculated for reactions where they cannot easily be measured.

If molecules are regarded as a system of mass points between which harmonic forces operate, it is possible to derive equations relating the vibration frequencies with the nuclear masses and force constants for stretching of bonds and bending of angles. Comparison of the force constant of the same linkage in different compounds will give a direct indication of the multiple or hybrid character of bonds. Interesting results have been obtained in this way for some relatively simple molecules. Progress is limited, however, not only by the severity of the computations required, but also by the more fundamental problem of selecting a valid function to express the variation of potential energy with nuclear configuration. Different types of force field have been assumed, and although no general rules can yet be laid dowi, the most satisfactory seems to be that which assumes in addition to valency forces certain interaction terms. Future progress must be guided by considering series of related molecules; and thus discovering which types of interaction are important.

As explained already, particular vibration frequencies will be permitted to appear either in the Raman spectrum or in the infra-red spectrum, in both, or in neither, according to the symmetry point group to which the molecule belongs. A comparison of the spectra may therefore fix the symmetry class, and hence decide between possible alternative molecular structures. Benzene and cyclohexane have been studied in this way. In the former case, the question is to decide whether the molecule has a centre of symmetry, which a resonance hybrid should show, but which the Kekulé structure does not have. In the latter case we have to decide between a planar, boat-shaped, or chair-shaped structure. Results are so far rather unconvincing, mainly because the Raman measurements are normally made with the liquids, where molecular distortions lead to a breakdown of the selection rules.

Although any molecular vibration in reality in. volves the whole molecule, some linkages retain individuality and give rise to characteristic absorption frequencies. Such linkages can therefore be detected or estimated in molecules. The absence of the characteristic O-H group absorption in some hydroxy compounds has proved the existence of the hydrogen bond and revealed unexpected cases of inter- and intra-molecular association. The individuality of linkages tends to disappear when the nuclei are roughly equal in mass or the force constants roughly equal in value. In spite of this, some nuclear skeletons, 
such as the nitro or tertiary butyl groups, retain characteristic sets of frequencies in different compounds. Deformational motions are also sometimes characteristic. For example, the symmetrical deformational frequency of the $\mathrm{CH}_{3}$-group can be used to indicate its presence in polythene; and different types of olefine can be distinguished by differences in some of their deformational vibrations. The latter results can be used to study the cracking of hydrocarbons or the method of polymerization of un. saturated compounds. Thus, when 1.3 butadiene condenses to form buna rubber, the extent of 1.2 or 1.4 addition can be estimated. In polymeric chemistry a variety of problems can be tackled successfully, such as the arrangements within a long chain, or the structure of interpolymers.

Since no two molecules, except a pair of optical isomers, have the same nuclear configuration, the fact that any molecular vibration involves the entire molecule implies that no two molecules will have a completely identical set of vibration frequencies. The infra-red spectrum will therefore be a fingerprint of the molecule. On this basis, many mixtures, sometimes of great complexity and not susceptible to other methods, can be analysed. The method is particularly suitable for isomeric mixtures. Even stereoisomers show different spectra. Analyses of this kind are rapid and require a very small amount of material. Certain technical difficulties still exist, but are rapidly being overcome, and the method has wide applicability in organic chemistry. Accurate measurements on absorption intensities may also lead to more detailed knowledge about the nature of chemical bonds.

\section{THE ANTIQUITY OF MAN IN AUSTRALIA}

\section{By PROF. F. WOOD JONES, F.R.S. University of Manchester}

A

LARGE part of a recent Memoir of the National Museum, Melbourne (No. 13, 1943), is devoted to the question of the authentic antiquity of certain human artefacts and skeletal remains that have been recorded from various parts of Australia over a long series of years. The available evidence in relation to each find is carefully analysed, and a judicial summing up leads, in most cases, to a verdict of 'not proven'.

This new survey has been undertaken by the director of the Museum, Mr. D. J. Mahony, as a consequence of the recent discovery of mineralized human remains near Keilor at the junction of Dry Creek and the Maribyrnong River in the neighbourhood of Melbourne. The skull was unearthed from undisturbed strata $18 \mathrm{ft}$. below the surface. The district in which the find was made shows the presence of three distinct river terraces. The terrace from which the skull was excavated is the highest of the series and is $45 \mathrm{ft}$. above the adjacent river bed, the other terraces being respectively at the $36-\mathrm{ft}$. and $27-\mathrm{ft}$. levels. Mahony's conclusion from a geological survey of the area is that the terraces "represent the eustatic rise of sea level during the Riss-Würm interglacial phase" and that "the skull and the terrace are evidently contemporaneous". The correlation of the European Riss-Würm period with happenings in southern Australia and Tasmania is made in the assurance that "most glaciologists consider that glacial and interglacial phases were contemporaneous in both hemispheres".

The circumstances of the finding of the skull in situ in the undisturbed face of a sand-pit seem to be well authenticated. The skull itself is mineralized and bears every evidence of being contemporary with the stratum from which it was removed. We may therefore claim that the Keilor skull is the first Australian human fragment the geological antiquity of which is definitely guaranteed by the circumstances of its finding and that, in the opinion of competent Australian geologists, it dates from the Riss-Würm interglacial phase of the Pleistocene period. We may consider its geological story as settled, subject only to possible minor differences of opinion among experts concerning the precise age of the deposit in which the skull was found.

The study of the human remains is, however, far from being completed. In the introductory paper by Mr. Mahony, it is said that "Two mineralised human skulls and some other bones were found". The actual finder of the skull mentioned "one fossilised limb bone and several other fragments" as being with the skull. $\mathrm{He}$ adds that "since then five pieces of another skull were found at the same level and about 6 feet distant from the first skull". Only one skull (lacking the mandible) is in the possession of the National Museum, and it is the only specimen dealt with in this publication.

The account of the skull is written by Dr. J. Wunderly and that of the palate and maxillary teeth by Dr. W. Adam. Neither of these accounts can be considered as being definitive descriptions of the characters of the skeletal remains, and it might have been well to limit the account of the skull to a simple statement of its characters and to have refrained from any suggestions as to racial affinities until the rest of the skeletal remains were available for examination. Dr. Wunderly's conclusions concerning the skull are that "it combines Australoid and Tasmanoid characteristics in about equal proportions". Further, he states that "the Australoid and Tasmanoid anatomical characters are consistent with the theory that the Australians had a bi-racial origin, and also with the supplementary theory that Australia was originally peopled by Negritos. The presence of characteristics of the two racial types is more important than their proportional relationship". I find it difficult to understand the meaning of this last statement. Presumably it means that the Australian race as we know it is compounded of two primary races. But if Dr. Wunderly means that these two primary races were what we now know as Australians (Dravidian or Pre-Dravidian Cymotrichi) and Tasmanians (Oceanic Ulotrichi) is not clear, for the peopling of Australia by Negritos (presumably Tasmanians) he relegates to a "supplementary theory". Should he, in fact, mean that the Australian race is compounded of a Pleistocene Australian-Tasmanian mixture, he must be prepared to admit that the Tasmanian race is equally a mixture of the same two elements. For if that is not admitted, explanation is needed as to how, when Australian and Negrito (Tasmanian) had formed a mixed race in continental Australia in Pleistocene times, the Tasmanians, with all their racial characters intact, were present in Tasmania on the arrival of the white man. Only two possible explanations seem to present themselves. The first is that it so happened that the Ulotrichous Negritos who passed from Australia to Tasmania chanced, in 